\title{
Effects of Chili Treatment on Gastrointestinal and Rectal Sensation in Diarrhea-predominant Irritable Bowel Syndrome: A Randomized, Double-blinded, Crossover Study
}

\author{
Satimai Aniwan and Sutep Gonlachanvit* \\ Gastrointestinal Motility Research Unit, Division of Gastroenterology, Department of Internal Medicine, Chulalongkom University, Bangkok, Thailand
}

\begin{abstract}
Background/Aims
Whether, chronic chili ingestion can desensitize transient receptor potential vanilloid type 1 receptors in gastrointestinal (GI) tract leading to decrease GI symptoms and sensation in diarrhea-predominant irritable bowel syndrome (IBS-D) patients has not been well explored. The aim of this study was to determine the effects of 6-week chili treatment on postprandial GI symptoms and rectal sensation in response to balloon distention in IBS-D patients.
\end{abstract}

\section{Methods}

Sixteen IBS-D patients received placebo or chili capsules before meals 3 times/day for 6 weeks in a randomized, double-blinded, crossover fashion with 4-week washout period. Postprandial Gl symptoms were evaluated. All patients underwent a rectal barostat study to evaluate rectal sensory threshold at the end of each treatment.

\section{Results}

The maximum postprandial abdominal burning scores were similar between both treatments at baseline $(1.4[0.0-2.0]$ vs. 1.1 $[0.0-2.8], P>0.05)$ but were significantly decreased after chili $(0.0[0.0-0.5]$ vs. $0.3[0.0-1.6], P<0.05)$ at the end of treatment. The chili treatment significantly increased sensory threshold for the first rectal sensation (median [interquartile range]:16 [12-16] $\mathrm{mmHg}$ vs. 8 [8-16] $\mathrm{mmHg}, P<0.05)$ however, there was no significant effect on rectal compliance (7.3 \pm 1.0 vs. $7.1 \pm 1.8 \mathrm{~mL} / \mathrm{mmHg}$ ). Other postprandial $\mathrm{Gl}$ symptoms did not vary significantly between both treatments at baseline and the end of treatment.

\section{Conclusions}

In IBS-D patients, 6-week chili ingestion significantly decreased postprandial abdominal burning and increased the rectal sensory threshold. These findings suggest a desensitization effect of chili ingestion on transient receptor potential vanilloid type 1 receptors in the proximal gut and rectum.

(J Neurogastroenterol Motil 2014;20:400-406)

Received: February 27, 2014 Revised: March 23, 2014 Accepted: March 27, 2014

(c) This is an Open Access article distributed under the terms of the Creative Commons Attribution Non-Commercial License (http://creativecommons. org/licenses/by-nc/3.0) which permits unrestricted non-commercial use, distribution, and reproduction in any medium, provided the original work is properly cited.

*Correspondence: Sutep Gonlachanvit, MD

Gastrointestinal Motility Research Unit, Division of Gastroenterology, Department of Internal Medicine, Faculty of Medicine, Chulalongkorn University, Rama 4 Road, Patumwan, Bangkok 10330, Thailand

Tel: +66-2-256-4265, Fax: +66-2-252-7839, E-mail: gsutep@hotmail.com

Financial support: None.

Conflicts of interest: None.

Author contributions: Sutep Gonlachanvit was responsible for the concept and study design, and reviewed this manuscript as a corresponding author; Satimai Aniwan performed data collection and statistical analysis, and wrote the full manuscript.

ORCID: Satimai Aniwan, http://orcid.org/0000-0002-2730-2450. 


\section{Key Words}

Capsaicin; Gastrointestinal tract; Irritable bowel syndrome; Sensation

\section{Introduction}

Visceral hypersensitivity has been considered a significant pathophysiology in irritable bowel syndrome (IBS). Capsaicin receptors, transient receptor potential vanilloid type 1 (TRPV1) receptors, are expressed in the gut and play a role in visceral nociception. ${ }^{1}$ Increased TRPV1 receptors was found in the gut mucosa of patients with functional gastrointestinal (GI) disorders; the esophagus of non-erosive reflux disease (NERD) patients, ${ }^{2}$ the colon of IBS patients ${ }^{3}$ and the rectum of rectal hypersensitivity patients. ${ }^{4}$

Capsaicin, is a pungent ingredient in chili, can stimulate TRPV1 receptors and mediate pain and burning sensation. ${ }^{5}$ In IBS patients, acute chili ingestion induces abdominal pain, burning and rectal hyperalgesia. ${ }^{6}$ A previous study demonstrated that acute ingestion of chili-containing meals produced higher abdominal pain and abdominal burning symptom than standard meals, and the increase in symptom severity was greater in diarrhea-predominant IBS (IBS-D) patients than healthy volunteers. ${ }^{7}$ These data suggest that chili can modulate both proximal and distal gut sensation in IBS patients. The chronic chili ingestion may desensitize TRPV1 receptors and improve in GI symptoms of functional dyspepsia ${ }^{8}$ and NERD patients ${ }^{9}$ However, the desensitization effect of chronic chili ingestion on TRPV1 receptors in IBS patients has not been clearly demonstrated.

The objective of this study was to determine the effect of 6-week chili ingestion on abdominal burning and pain that were induced by spicy meals and to assess rectal sensation in response to balloon distention in IBS-D patients.

\section{Materials and Methods}

\section{Patients}

A total of 16 IBS-D patients (11 female; age, $46 \pm 3$ years) who fulfilled the Rome III criteria were recruited from August 2010 to August 2012. All IBS-D patients had a normal colonoscopy at least 1 year before enrollment. Patients with diabetes mellitus, major neurological disorders, psychological disorders, pregnancy and history of abdominal surgery or severe GI symptoms after consuming spicy foods were excluded. Medications that affected GI motility and sensation; anti-diarrheals, anticholinergics, smooth muscle relaxants and domperidone were stopped at least 7 days before study. All patients were asked to avoid chili and spicy foods at least 7 days before study and during study. Due to previous study suggested that the desensitization effect of capsaicin on TRPV1 receptors recovered completely within 3 days after withdrawal of the capsaicin stimulation. ${ }^{10}$ The study was approved by the institutional review board of the Faculty of Medicine, Chulalongkorn University. All patients provided written informed consent.

\section{Study Design}

All patients were randomized to receive either chili (1 gelatin and 1 enteric-coated capsule) or a placebo in capsules 3 times/day for 6 weeks in a randomized, double-blind, crossover manner.

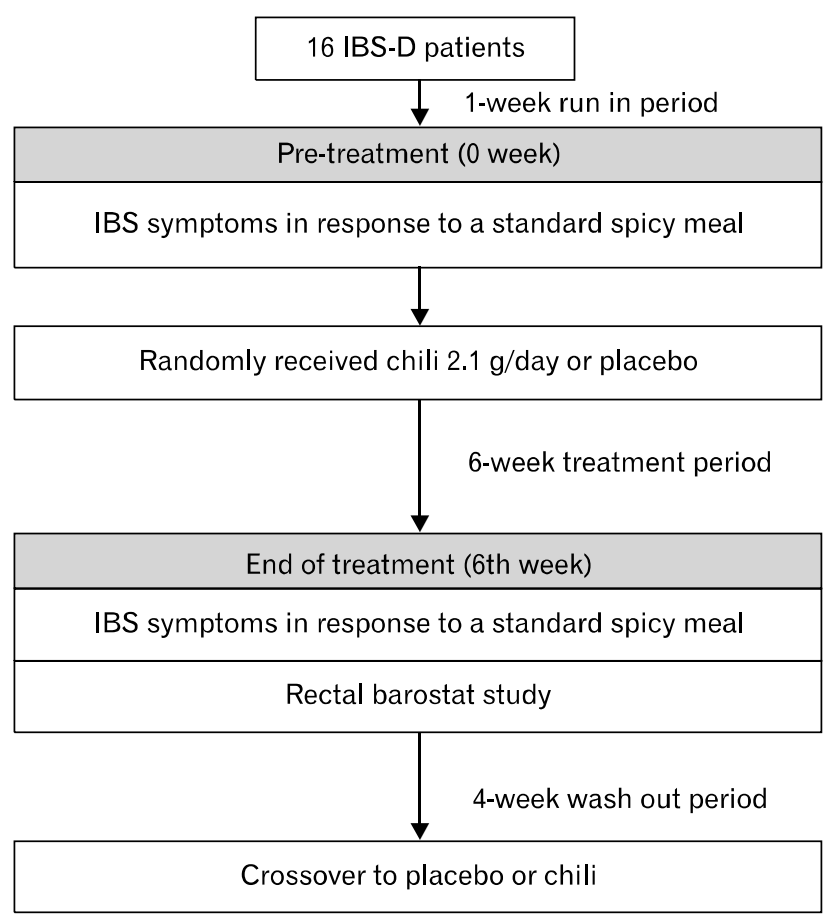

Figure 1. Study flow diagram. IBS, irritable bowel syndrome; IBS-D, diarrhea-predominant irritable bowel syndrome. 
There was a 4-week washout period. Patients were asked to take the capsules 15 minutes before meal. To increase the availability of capsaicin at the distal GI tract, we prepared gelatin and enteric-coated capsules. The gelatin capsule was prepared to be dispersed in the stomach. Each gelatin capsule contained $0.5 \mathrm{~g}$ red chili powder (Capsicum frutescens Linn, $1.2 \mathrm{mg}$ capsaicin/1 g dry weight; Nguan Soon Co., Ltd., Bangkok, Thailand). The enteric-coated capsule was designed to be dispersed in the small intestine. Each capsule contained $0.2 \mathrm{~g}$ chili mixed with $0.3 \mathrm{~g}$ lactose. Either gelatin or enteric-coated capsule contained $0.5 \mathrm{~g}$ lactose powder, which was used as a placebo. Total dose of chili $(2.1 \mathrm{~g} /$ day) corresponded to the amount of chili in typical single-serving Thai meal. The average chili consumption in a Thai person is $5 \mathrm{~g} /$ day. $^{11}$

The GI symptoms in response to a standard spicy meal were evaluated at baseline and at the end of both treatments. To determine the effect of chronic chili ingestion on rectal sensation, all patients underwent a rectal barostat study at the end of both treatments (Fig. 1).

\section{Evaluation of Gastrointestinal Symptoms in Response to Standard Spicy Meals}

To evaluate severity of GI symptoms in response to standard spicy meal, the symptoms were assessed 5 minutes before spicy meal, immediately after meal and every 15 minutes for 2 hours using $10 \mathrm{~cm}$ long visual analogue scales ${ }^{7}$ before and at the end of both treatments. The symptoms were evaluated: (1) abdominal pain, (2) abdominal burning, (3) bloating and (4) postprandial fecal urgency. The standard spicy meal was ingested in the morning after an overnight fast. The evaluation of GI symptoms in response to spicy meal at the end of the treatment period was performed 30 minutes after rectal barostat study.

\section{Rectal Barostat Study}

A rectal barostat study was performed at the end of each treatment to determine the effect of chronic chili on rectal sensation compare with placebo. The barostat study was performed using a polyvinyl catheter that was incorporated with a $500 \mathrm{~mL}$ non-compliance polyethylene bag, which was inflated by a 2.8 $\mathrm{mm}$ central lumen and an electronic barostat (Distender Series II; G\&J Electronics Inc., Toronto, Canada). The barostat bag was placed $5-15 \mathrm{~cm}$ from the anal verge. Then, the bag was inflated with $120 \mathrm{~mL}$ of air and deflated completely. After 5-minute rest period, the perception and pressure-volume relationship in response to rapid phasic distention was studied by applying stepwise pressure $(8,12,16,20,24,28,32,36,40,44$ and 48 $\mathrm{mmHg}$ ). The bag was distended at $40 \mathrm{~mL} / \mathrm{sec}$ for 1 minute with a 1-minute interval to allow the intrabag pressure to reduce to 0 $\mathrm{mmHg}$.

Rectal sensation of stool perception was scored $0-4(0=$ no sensation, 1 = first sensation of stool, 2 = first sensation of urgency of stool, $3=$ moderate urgency of stool and $4=$ severe urgency of stool with intolerable need to pass stool). Patients were asked to record rectal sensation 50 seconds after the onset of each distension. ${ }^{12}$ Pressure threshold was defined as the minimum pressure that induced the first perception of each sensation.

\section{Evaluation of Gastrointestinal Symptoms in Response to the Chili and Placebo Treatments}

The GI symptoms and adverse events were evaluated at baseline, the first, second, fourth and sixth week of treatment. Using visual analogue scales, patients were asked to rate their symptoms within previous 7 days. The GI symptoms were evaluated; abdominal pain, abdominal burning, bloating, postprandial fecal urgency, diarrhea, incomplete evacuation, global GI symptom and interrupted normal daily activity. Stool patterns were assessed using Bristol Stool Form Scale. An antacid gel was prescribed as a rescue medication in case severe abdominal pain or burning. Unused capsules were counted to evaluate compliance.

\section{Statistical Methods}

The data were expressed as the mean \pm SEM or the median and interquartile range. The Kolmogorov-Smirnov test was used to determine the normality of the data distribution. Comparisons of the parametric data were analyzed using a paired Student's $t$ test. Comparisons of the non-parametric data were analyzed using Wilcoxon's signed rank test. The primary endpoints were abdominal burning and abdominal pain symptoms induced by standard spicy meal and rectal sensation in response to balloon distention. A sample size of 16 patients per group was needed to achieve $80 \%$ power for the detection of the differences in abdominal burning scores between 2 treatments. A $P$-value $<0.05$ was considered statistically significant.

\section{Results}

All 16 patients completed study without serious adverse events. Abdominal warmth transiently developed within an hour after chili ingestion in 4 patients but only in 1 patient after 
placebo. The mean duration of IBS was $4.6 \pm 1.3$ years. The baseline GI symptoms before each treatment are summarized in Table. There were no significant differences between the 2 treatments according to GI symptoms and global GI symptoms at baseline. Eight patients were randomized to receive chili first and 8 patients received placebo first.

\section{Effect of Chronic Chili Ingestion on Gastrointestinal Symptoms in Response to Standard Spicy Meal}

At baseline, the maximum abdominal burning, abdominal pain, and other GI symptom scores in response to spicy meal were not significantly different between 2 treatments $(P>0.05)$. At the end of treatment, the maximum abdominal burning scores, but not abdominal pain scores were significantly decreased after chili treatment compared with placebo $(P<0.05)$ (Fig. 2). At the end of treatment, the maximum bloating scores, were lower in chili treatment compared with placebo; however, the differences were not statistically significant $(P>0.05)$.

\section{Effect of Chronic Chili Ingestion on Rectal Sensation in Response to Rectal Balloon Distention}

At the end of the treatment, chronic chili ingestion significantly increased sensory threshold for the first rectal sensation (median [interquartile range]; 16 [12-16] vs. 8 [8-16] $\mathrm{mmHg}$, $P<0.05)$, and there was a trend toward increased sensory threshold in chili treatment for the first $(20$ [16-20] vs. 16

Table. Baseline Gastrointestinal Symptoms Within the Previous 7 Days Before the Chili and Placebo Treatments $(P>0.05)$

\begin{tabular}{lcc}
\hline & Chili & Placebo \\
\hline Abdominal pain & $2.5(0.3-5.0)$ & $2.0(0.0-4.0)$ \\
Abdominal burning & $1.5(0.0-5.0)$ & $0.0(0.0-5.8)$ \\
Abdominal bloating & $2.0(0.9-7.0)$ & $2.0(0.0-4.5)$ \\
Postprandial fecal urgency & $3.2(1.1-7.5)$ & $2.5(0.0-5.2)$ \\
Diarrhea & $0.8(0.0-5.6)$ & $0.0(0.0-4.8)$ \\
Incomplete evacuation & $1.0(0.0-6.0)$ & $1.3(0.0-4.5)$ \\
Global gastrointestinal symptom & $4.7(2.0-6.0)$ & $5.0(2.0-8.0)$ \\
Interrupted normal daily activity & $7.0(2.6-9.0)$ & $3.4(1.8-9.0)$ \\
Stool frequency per day & $4(3-6)$ & $3(2-5)$ \\
Stool consistency (BSFS 1-7) & $5(4-6)$ & $5(4-6)$ \\
\hline
\end{tabular}

BSFS, Bristol Stool Form Scale.

Values are median (interquartile range).

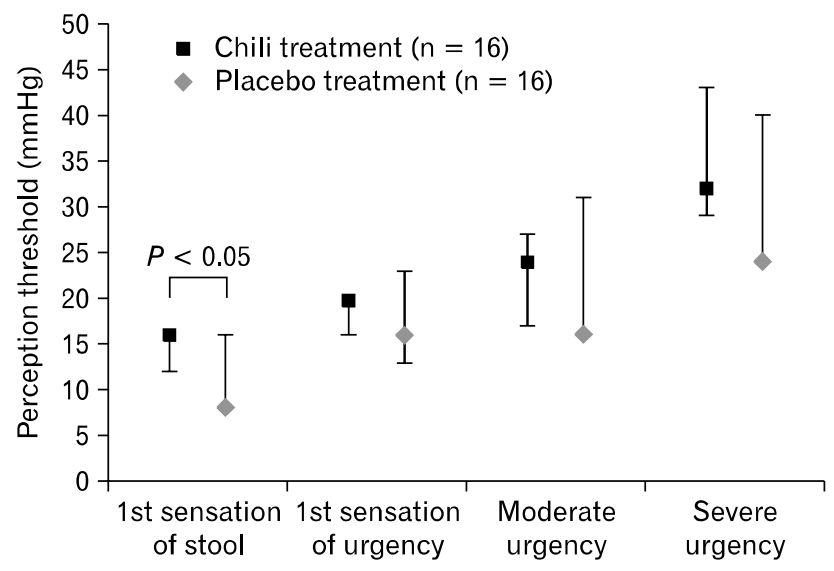

Figure 3. Rectal sensation at the end of chili and placebo treatments.

A

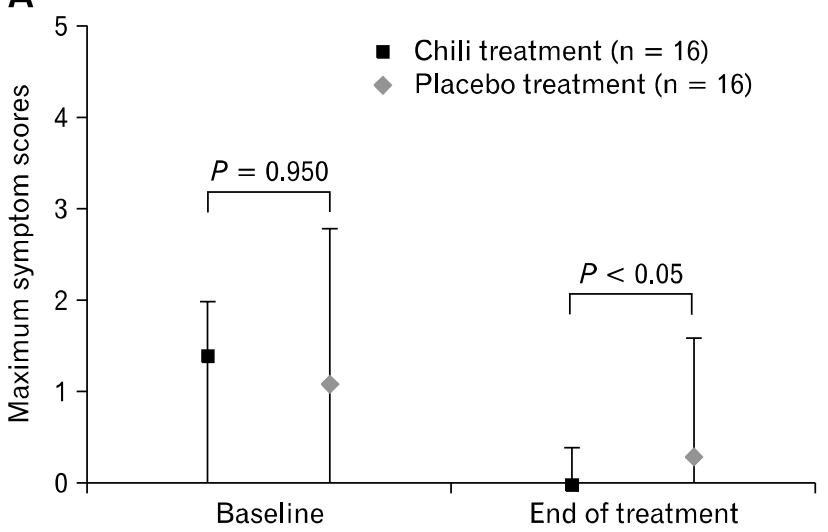

B

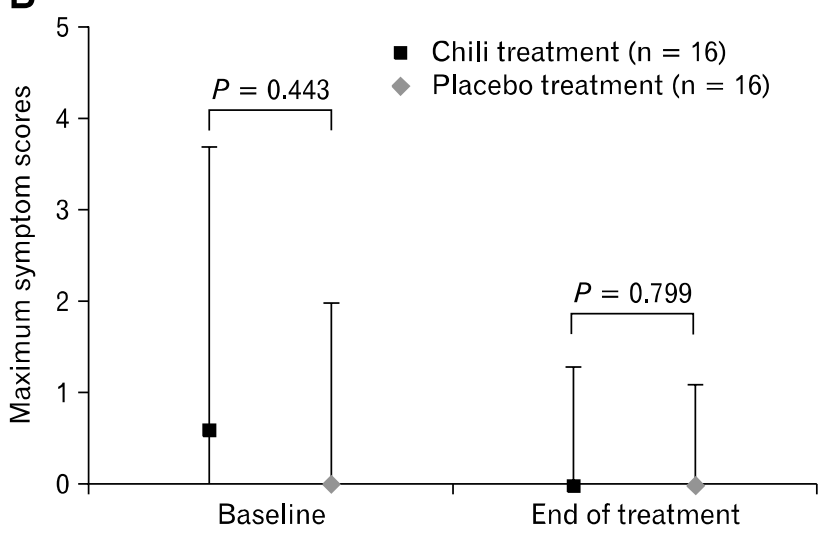

Figure 2. Effect of chronic chili ingestion on postprandial gastrointestinal symptoms. (A) Abdominal burning symptoms in response to the spicy meal at baseline and at the end of chili and placebo treatments. (B) Abdominal pain symptoms in response to the spicy meal at baseline and at the end of chili and placebo treatments $(P>0.05)$. 
A

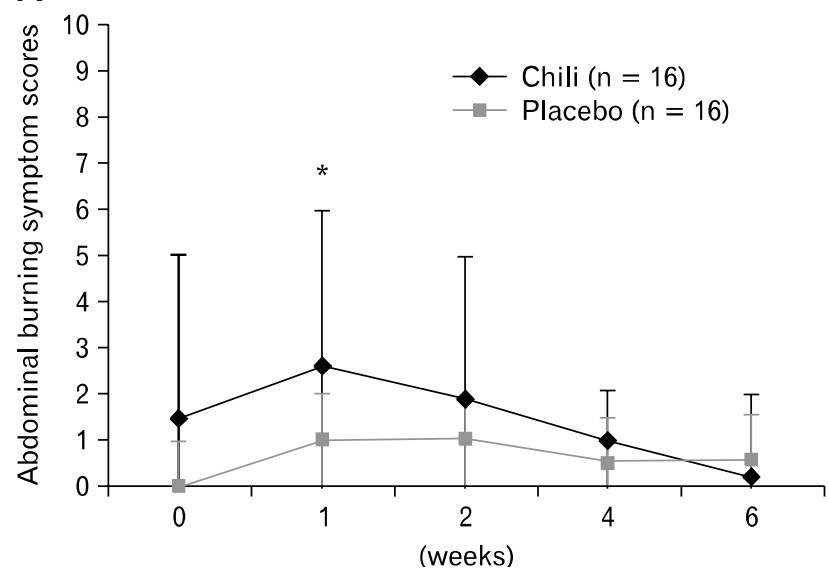

B

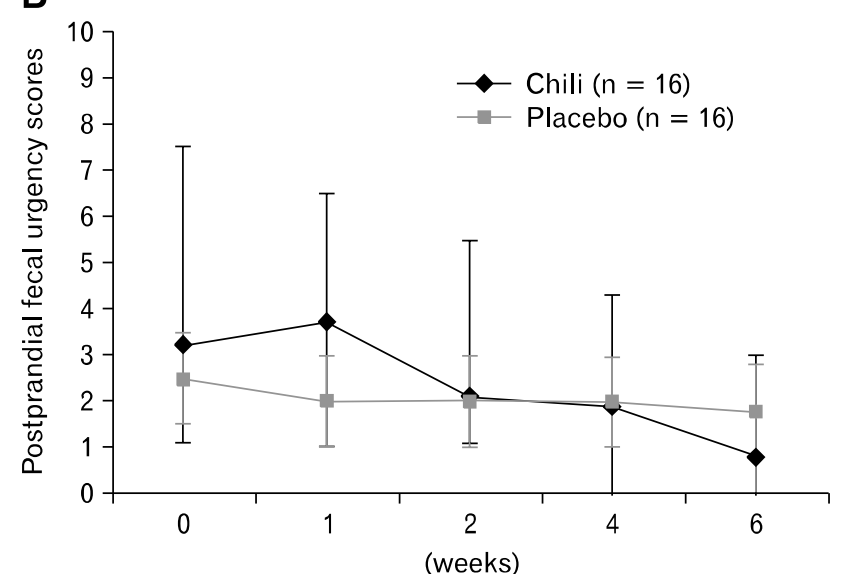

Figure 4. The mean gastrointestinal symptoms during 6-week chili and placebo treatments. (A) The mean abdominal burning scores within the previous 7 days before each symptom evaluation during chili and placebo treatments $\left({ }^{*} P<0.05\right)$. (B) The mean postprandial fecal urgency scores within previous 7 days before each symptom evaluation during chili and placebo treatments.

[13-23]), moderate (24 [17-27] vs. 16 [16-31]) and severe urgency (32 [29-43] vs. 24 [24-40]) compared with placebo (Fig. $3)$. No significant differences in rectal compliance were observed between chili and placebo treatment $(7.3 \pm 1.0$ vs. $7.1 \pm 1.8$ $\mathrm{mL} / \mathrm{mmHg} ;>0.05)$.

\section{Effect of Chili Treatment on Gastrointestinal Symptoms in Diarrhea Predominant Irritable Bowel Syndrome Patients}

The baseline GI symptom scores were similar between 2 treatments $(P>0.05)$. After chili treatment, abdominal burning and postprandial fecal urgency transiently increased during the first and second week (Fig. 4). At the end of treatment, abdominal burning and postprandial fecal urgency scores were lower in chili treatment than placebo, but did not reach statistically significance (Fig. 4). There were no any significant differences of the other symptoms scores after chili treatment and placebo treatment with respect to pre-treatment. The other symptom scores did not indicate any significant differences between chili and placebo treatment.

\section{Discussion}

This study demonstrated 6-week chili ingestion significantly reduced spicy meal induced abdominal burning symptoms compared with placebo. Chili significantly increased sensory threshold of perception for the first rectal sensation in response to balloon distention without significant effect on rectal compliance in
IBS-D patients. These suggest that chili treatment used in this study can modify sensations of the proximal gut and rectum.

As a result of acute chili exposure to gut mucosa, it was associated with enhanced warm or burning sensation mediated by TRPV1 receptors. ${ }^{7,13}$ Subsequently, chronic chili ingestion decreased in burning symptoms in response to standard spicy meal these suggests a desensitization effect. Similar to previous studies showed chronic chili ingestion can modify symptoms in other functional GI diseases. ${ }^{8,9,14}$ Bortolotti et $\mathrm{al}^{8}$ showed 5 -week red pepper (2.5 g/day) decreased epigastric pain, fullness and nausea in 30 functional dyspepsia patients. In a preliminary study by Jutaghokiat et al, ${ }^{9}$ found that 6-week chili (3 g/day) can improve heart burn and regurgitation symptoms in 8 NERD patients.

Meanwhile previous studies in IBS patients demonstrated acute chili ingestion can increase abdominal burning, pain and rectal sensation. ${ }^{6,7}$ Later, Bortolotti et $\mathrm{al}^{14}$ studied the effect of chronic red pepper $(0.6 \mathrm{~g})$ in IBS patients. The results revealed chronic red pepper significantly decreased abdominal pain and bloating with respect to pre-treatment but did not to placebo. However, 8 patients were dropped from the study, and 8 patients needed the $50 \%$ dose reduction of red pepper because of abdominal pain. ${ }^{14}$

Although visceral hypersensitivity has been observed in IBS, IBS-D had more rectal hypersensitivity than constipation-predominant IBS (IBS-C). ${ }^{1,15}$ In contrast, IBS-C patients were reported to be rectal hyposensitive. ${ }^{15}$ Thus, we decided to study the IBS-D subtype. Our results demonstrated abdominal burning and fecal urgency transiently increased during the first and sec- 
ond weeks of chili treatment and gradually decreased over time compared with placebo. Additionally, chili treatment decreased rectal sensation in response to balloon distention. These results suggest the repeated exposure of gut mucosa to chili induced sensitization at the beginning, followed by desensitization of the capsaicin receptors in the proximal gut and rectal. However we could not demonstrate an improvement in IBS symptoms. This may be explained by (1) the dose of chili may be not high enough. Since the optimum dose of chili is unknown the stepwise dosage should be considered and (2) the small sample size of the patients in this study, which was designed to determine the sensation changes in response to the spicy meal and rectal balloon distention. Therefore a larger randomized control trial is needed to confirm whether chili ingestion can improve IBS symptoms.

Whereas our results showed chili decreased abdominal burning induced by spicy meal, but not abdominal pain suggests a different pathophysiology of burning and pain. A previous study demonstrated that chemical stimulation in the proximal small intestine by capsaicin induced a greater severity of burning symptoms than mechanical stimulation by balloon distention; however, a similar severity of abdominal pain was induced in response to balloon distention. ${ }^{16}$

Because capsaicin is small lipophilic that is rapidly absorbed. ${ }^{16}$ The different parts of human gut incur different capsaicin dose effects. The duodenal capsaicin-induced sensations occur at lower doses than jejunum. ${ }^{16}$ Therefore, the capsaicin dose, form and dispersed site should be considered. In our study, we administered chili in enteric-coated capsules to avoid a low capsaicin level in the small intestinal and colonic lumen. Chili in gelatin capsules was administered to ensure the availability of capsaicin throughout the whole gut. Although, a single dose of $5 \mathrm{~g}$ chili for 3 days has been reported to affect rectal sensation, ${ }^{13}$ this high dose cannot be tolerated by most IBS patients.

There were several limitations in this study. Most patients experienced mild abdominal burning after chili ingestion; therefore, they were incompletely blinded. Using chili as IBS treatment may not be applicable in populations that irregularly consume spicy meals because this treatment may induce more adverse symptoms, especially abdominal burning and pain, compared with our Thai patients. A gradual increase in chili dose may be helpful.

There has been increasing evidence to support the role of desensitization of capsaicin receptors for functional GI disorder including functional dyspepsia and IBS. These results support the use of chili, natural capsaicin receptor agonist for the treatment of
IBS symptoms. However, further research studies are warranted to confirm this hypothesis.

In conclusion, chronic chili ingestion significantly reduced abdominal burning, which was induced by standard spicy meal and rectal sensation in response to balloon distention in IBS-D patients. These results suggest a desensitization effect of chronic chili ingestion on capsaicin receptors in the GI tract in IBS-D patients and support the use of capsaicin agonists for the treatment of IBS symptoms.

\section{References}

1. Delvaux M. Role of visceral sensitivity in the pathophysiology of irritable bowel syndrome. Gut 2002;51(suppl 1):i67-i71.

2. Bhat YM, Bielefeldt K. Capsaicin receptor (TRPV1) and non-erosive reflux disease. Eur J Gastroenterol Hepatol 2006;18:263-270.

3. Akbar A, Yiangou Y, Facer P, Walters JR, Anand P, Ghosh S. Increased capsaicin receptor TRPV1-expressing sensory fibres in irritable bowel syndrome and their correlation with abdominal pain. Gut 2008;57:923-929.

4. Chan CL, Facer P, Davis JB, et al. Sensory fibres expressing capsaicin receptor TRPV1 in patients with rectal hypersensitivity and faecal urgency. Lancet 2003;361:385-391.

5. Camilleri M, Coulie B, Tack JF. Visceral hypersensitivity: facts, speculations, and challenges. Gut 2001;48:125-131.

6. Schmulson MJ, Valdovinos MA, Milke P. Chili pepper and rectal hyperalgesia in irritable bowel syndrome. Am J Gastroenterol 2003;98:1214-1215.

7. Gonlachanvit S, Mahayosnond A, Kullavanijaya P. Effects of chili on postprandial gastrointestinal symptoms in diarrhoea predominant irritable bowel syndrome: evidence for capsaicin-sensitive visceral nociception hypersensitivity. Neurogastroenterol Motil 2009;21:23-32.

8. Bortolotti M, Coccia G, Grossi G, Miglioli M. The treatment of functional dyspepsia with red pepper. Aliment Pharmacol Ther 2002;16:1075-1082.

9. Jutaghokiat S, Imraporn B, Gonlachanvit S. Chili improves gastroesophageal reflux symptoms in patients with non erosive gastroesophageal reflux disease (NERD). Gastroenterology 2009;136(suppl 1):A92.

10. Karrer T, Bartoshuk L. Effects of capsaicin desensitization on taste in humans. Physiol Behav 1995;57:421-429.

11. Interdepartment Commitee on Nutrition for National Defense. Nutrition survey - The kingdom of Thailand. Washington DC: US Goverment Printing Office 1962:57-59.

12. Gonlachanvit S, Rhee J, Sun WM, Chey WD. Effect of acute acoustic stress on anorectal function sensation in healthy human. Neurogastroenterol Motil 2005;17:222-228.

13. Gonlachanvit S, Fongkam P, Wittayalertpanya S, Kullavanijaya P. Red chili induces rectal hypersensitivity in healthy humans: possible role of 5HT-3 receptors on capsaicin-sensitive visceral nociceptive pathways. Aliment Pharmacol Ther 2007;26:617-625.

14. Bortolotti M, Porta S. Effect of red pepper on symptoms of irritable bowel syndrome: preliminary study. Dig Dis Sci 2011;56:3288- 
3295 .

15. Harraf F, Schmulson M, Saba L, et al. Subtypes of constipation predominant irritable bowel syndrome based on rectal perception. Gut 1998;43:388-394.
16. Hammer J, Vogelsang H. Characterization of sensations induced by capsaicin in the upper gastrointestinal tract. Neurogastroenterol Motil 2007;19:279-287. 